OPEN ACCESS

Edited by:

Qiang Huang,

Beijing Institute of Technology, China

Reviewed by:

Yang Shi,

University of Victoria, Canada

Tara Julia Hamilton,

Western Sydney University, Australia

*Correspondence:

Feihu Zhang

feihu.zhang@nwpu.edu.cn

Received: 12 October 2016

Accepted: 23 June 2017

Published: 12 July 2017

Citation:

Li H, Liu M and Zhang F (2017)

Geomagnetic Navigation of

Autonomous Underwater Vehicle Based on Multi-objective Evolutionary

Algorithm. Front. Neurorobot. 11:34.

doi: 10.3389/fnbot.2017.00034

\section{Geomagnetic Navigation of Autonomous Underwater Vehicle Based on Multi-objective Evolutionary Algorithm}

\author{
Hong Li, Mingyong Liu and Feihu Zhang * \\ School of Marine Science and Technology, Northwestern Polytechnical University, Xi'an, China
}

This paper presents a multi-objective evolutionary algorithm of bio-inspired geomagnetic navigation for Autonomous Underwater Vehicle (AUV). Inspired by the biological navigation behavior, the solution was proposed without using a priori information, simply by magnetotaxis searching. However, the existence of the geomagnetic anomalies has significant influence on the geomagnetic navigation system, which often disrupts the distribution of the geomagnetic field. An extreme value region may easily appear in abnormal regions, which makes AUV lost in the navigation phase. This paper proposes an improved bio-inspired algorithm with behavior constraints, for sake of making AUV escape from the abnormal region. First, the navigation problem is considered as the optimization problem. Second, the environmental monitoring operator is introduced, to determine whether the algorithm falls into the geomagnetic anomaly region. Then, the behavior constraint operator is employed to get out of the abnormal region. Finally, the termination condition is triggered. Compared to the state-of- the-art, the proposed approach effectively overcomes the disturbance of the geomagnetic abnormal. The simulation result demonstrates the reliability and feasibility of the proposed approach in complex environments.

\section{Keywords: geomagnetic navigation, bio-inspired navigation, geomagnetic anomaly, local optimal, evolutionary} algorithm

\section{INTRODUCTION}

Autonomous Underwater Vehicle (AUV) has been widely used for both civilian and military applications, such as laying pipelines, ocean data collection, underwater equipment maintenance, and laying mines (Wadhams, 2012; Wynn et al., 2014; Shi et al., 2017). Most navigation systems rely on dead-reckoning that is by using inertial navigation system and velocity information from the Doppler sonar. However, the cumulative error becomes difficult to handle during the navigation, in contrast to the GPS and the acoustic transponder networks (Caiti et al., 2014).

Various techniques have been developed to eliminate the dead-reckoning errors (Hao et al., 2008; Yi et al., 2008; Shen et al., 2017). The geomagnetic navigation, which is originated from animal behaviors due to the earth's magnetic fields, has been widely used (Gould, 1984; Lohmann, 2010). It overcomes the following drawbacks: the error accumulation from the inertial navigation system, and the rapid attenuation signal from the satellite navigation system (Goldenberg, 2006). Thus, the geomagnetic field plays a key role in navigation, as it provides the position information in large scale 
environments (Teixeira and Pascoal, 2008). Typically, each point on the near-earth space has an unique magnetic field vector with respect to the corresponding coordinate, while the geomagnetic navigation is employed to provide a reliable navigation reference (Fu-qing, 2006; Zhou et al., 2008).

Based on the characteristics of the geomagnetism, numbers of methods have been developed (Caifa et al., 2011). The principle idea is employed based on the matching algorithms, such as MSD (Mean Square Difference), MAD (Mean Absolute Difference), ICCP (Iterative Closest Contour Point Algorithm) (Jia et al., 2012; Xie et al., 2013; Chong et al., 2014). However, traditional matching algorithms strongly depend on a priori geomagnetic map, which is quite challenging to acquire in practice.

To address this issue, the bio-inspired geomagnetic navigation method was proposed from animal behaviors (Liu et al., 2013). The most informative experimental paradigms have verified that the animals (like sea turtles and pigeons) can geomagnetic navigation to reach their goal locations (Paolo et al., 2003). Schulten concluded that animals can navigate by relying on geomagnetic sensitivity (Schulten, 1982). Winklhofer proposed the magneto-reception mechanisms to explain animals' navigation behavior (Winklhofer, 2009). Mole rats and salmons are also sensitive to the earth's magnetic field (Kimchi et al., 2004; Hays, 2013). In conclusion, the bio-inspired geomagnetic navigation provides a natural solution for navigation problem without a priori geomagnetic map.

However, the geomagnetic anomaly often influences the bioinspired geomagnetic navigation system which is caused by large iron ores. Boström showed that many geomagnetic blind spots were existed with respect to some migrating animals (Boström et al., 2012). Kiliowska pointed out the geomagnetic anomalies have interfered the whales' navigation system and caused them to beach (Klinowska, 1986). Lohmann pointed out the geomagnetic anomalies made the migrating turtles lost into Mexico (Lohmann et al., 2007). Dennis pointed out the pigeons were also easily lost when they were released in the geomagnetic anomaly area (Dennis et al., 2007). With respect to an AUV navigation, the geomagnetic anomalies disrupt the distribution of the geomagnetic field, which makes AUV lost in the navigation phase. In our previous works, the bio-inspired navigation algorithm was easily trapped in a local minimum point, caused by the geomagnetic anomalies (Liu et al., 2014). This happens where the distribution of the multiple geomagnetic was changed to the unsmooth and discontinuous regions ("concave" or "convex"). This caused the multi-objective search to converge to a local minimum easily (see Figure 1).

This paper presents a multi-objective evolutionary algorithm to address the local minimum problem by using the behavior constraint strategy. Main contributions are summarized as follows: First, it determines whether the algorithm is falling into the abnormal area. Second, it provides the strategy to jump out of the geomagnetic anomaly area. Once the algorithm is trapped in the local minimum, the behavior constraints are utilized to jump out the abnormal area.

The rest of this paper is organized as follows: Section Problem Formulation briefly introduces both the multi-objective search and the local minimum problems, respectively. Section

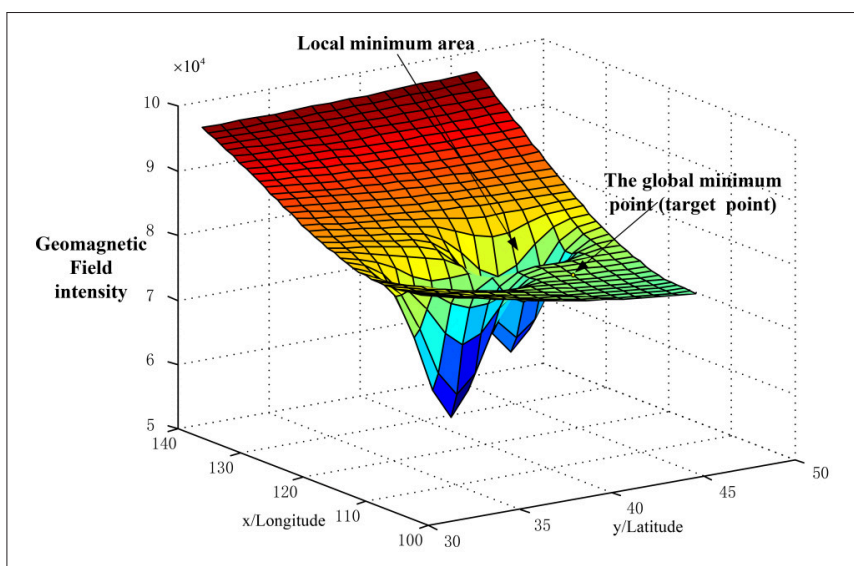

FIGURE 1 | The geomagnetic anomaly scenario.

Multi-Objective Evolutionary Algorithm with Geomagnetic Anomaly Presents the Multi-Objective Evolutionary Algorithm in Scenarios of Geomagnetic Anomaly. Section Numerical Simulations gives the main results and theoretical analysis. Finally, the conclusion is given in Section Conclusion.

\section{PROBLEM FORMULATION}

In this paper, the motion of AUV on the horizontal plane is considered in the $2 \mathrm{D}$ Cartesian coordinate system. This simplification is justified due to the fact that the difference of the geomagnetic in vertical is often negligible. Therefore, the kinematic equations of the motion are introduced as follows:

$$
\left\{\begin{array}{l}
x(k)=x(k-1)+v(k) \Delta T \cos \theta(k) \\
y(k)=y(k-1)+v(k) \Delta T \sin \theta(k)
\end{array}\right.
$$

where $\Delta T$ is the sample period, $k$ is the time instant, $v(k)$ is the kinematic velocity, $\theta(k)$ is the heading of AUV in a time instant. Here, assuming AUV moves with a constant velocity, formula (1) can be written as follows:

$$
\left\{\begin{array}{l}
x(k)=x(k-1)+L \cos \theta(k) \\
y(k)=y(k-1)+L \sin \theta(k)
\end{array}\right.
$$

where $L$ is the step size, $L=v(k) \Delta T$.

\section{Mathematical Description of the Multi-Objective Search Problem}

The geomagnetic fields include multiple geomagnetic components (Caifa et al., 2011), which can be described as follows:

$$
B=\left\{B_{1}, B_{2}, \cdots, B_{n}\right\}
$$

where $B$ is the set of the geomagnetic components vector, $B_{1}, B_{2}, \cdots, B_{n}$ are defined as geomagnetic components, such as the north magnetic field $B_{x}$, the east magnetic field $B_{y}$, the downward magnetic field $B_{z}$, the total intensity $B_{F}$, the horizontal magnetic field $B_{H}$, the declination angle $B_{D}$, and the declination angle $B_{I}$. 
The bio-inspired navigation is thus the convergence process of the geomagnetic components from the current position to the target position (Liu et al., 2014). Therefore, the navigation process can be considered as the multi-objective searching problem as follow:

$$
\begin{aligned}
& \left\{\begin{aligned}
\min F_{k}\left(f_{1}^{k}(B), f_{2}^{k}(B), \cdots, f_{n}^{k}(B)\right) & \begin{array}{l}
\text { Combining formulas (7) and (8), the convergence condition is } \\
\text { given by: }
\end{array} \\
\text { s.t. } f_{i}^{k}(B)=\left(B_{i}^{t}-B_{i}^{k}\right)^{2}, i \in n & \\
\left\|f_{i}^{\prime k}-f_{i}^{\prime k-1}\right\| & =\left\|\left(B_{i}^{t}+\Delta B^{k}-B_{i}^{k}\right)^{2}-\left(B_{i}^{t}+\Delta B^{k-1}-B_{i}^{k-1}\right)^{2}\right\| \\
& =\left\|\left(B_{i}^{t}-B_{i}^{k}\right)^{2}-\left(B_{i}^{t}-B_{i}^{k-1}\right)^{2}+2\left(B_{i}^{t}-B_{i}^{k}\right) \Delta B^{k}-2\left(B_{i}^{t}-B_{i}^{k-1}\right) \Delta B^{k-1}+\left(\Delta B^{k}\right)^{2}-\left(\Delta B^{k-1}\right)^{2}\right\| \\
& \leq\left\|\left(B_{i}^{t}-B_{i}^{k}\right)^{2}-\left(B_{i}^{t}-B_{i}^{k-1}\right)^{2}\right\|+\left\|2\left(B_{i}^{t}-B_{i}^{k}\right) \Delta B^{k}-2\left(B_{i}^{t}-B_{i}^{k-1}\right) \Delta B^{k-1}+\left(\Delta B^{k}\right)^{2}-\left(\Delta B^{k-1}\right)^{2}\right\| \\
& <\varepsilon+\left\|2\left(B_{i}^{t}-B_{i}^{k}\right) \Delta B^{k}-2\left(B_{i}^{t}-B_{i}^{k-1}\right) \Delta B^{k-1}\right\|+\left\|\left(\Delta B^{k}\right)^{2}-\left(\Delta B^{k-1}\right)^{2}\right\| \\
& =\xi
\end{aligned}\right.
\end{aligned}
$$

where $B_{i}^{t}$ is the geomagnetic component of the target position; $B_{i}^{k}$ is the geomagnetic component of the current position; $f_{i}^{k}$ is the difference of the $i^{\text {th }}$ geomagnetic component between the target position and the current position; $F_{k}$ is the objective function.

Considering the different magnitude of the geomagnetic components, the objective function is normalized as follows:

$$
F_{k}=\sum_{i=1}^{n} \frac{f_{i}^{k}(B)}{f_{i}^{0}(B)}=\sum_{i=1}^{n} \frac{\left(B_{i}^{t}-B_{i}^{k}\right)^{2}}{\left(B_{i}^{t}-B_{i}^{0}\right)^{2}}
$$

The errors between the current position and the target position can be assumed as the geomagnetic trend, while the searching process is terminated when the error converges to $\varepsilon$. This is expressed as:

$$
\lim _{k \rightarrow \infty}\left\|F_{k}-F_{k-1}\right\|<\varepsilon
$$

where $\varepsilon$ is a fixed value.

The bio-inspired navigation is thus considered as a posteriori searching problem, in presence of unknown geomagnetic components. Once the geomagnetic error between the current position and the target position converges to zero, the navigation process is terminated.

\section{Geomagnetic Searching in Anomaly Environment}

The geomagnetic anomaly field has significant influences on the geomagnetic navigation system, where the related factors are analyzed according to the distribution characteristics of the geomagnetic field (Talwani, 1965). Usually the geomagnetic anomaly is given by:

$$
B^{\prime}=B+\Delta B
$$

where $B$ is the amplitude of the abnormal region. where $\xi$ is bigger than $\varepsilon$ if the geomagnetic anomalies exists. This result shows that the algorithm is easily trapped in the local minimization.

\section{MULTI-OBJECTIVE EVOLUTIONARY ALGORITHM WITH GEOMAGNETIC ANOMALY}

This section gives the general idea of the proposed algorithm in geomagnetic anomaly, the experiment will be given in next section.

\section{The Search Principle}

A bio-inspired navigation method is investigated, which is based on a simple assumption that a homing animal only senses and compares the variation of the geomagnetic field to reach its home.

Evolutionary algorithm is often used to solve problems in highly complex spaces (Cliff et al., 1993; Droste et al., 2002; Vrugt and Robinson, 2007; Peng et al., 2016; Peng and Wu, 2017). Based on Darwin's evolutionary theory, ethologists have been concerned with the evolution of animals' behavior (Alerstam et al., 2003). Searching behavior can be described as active movement, in which animals attempt to find resources such as food, mates, nesting sites (Bell, 1992). Based on this, McFarland has proposed a number of mathematical analogs by using adaptive evolutionary behaviors (McFarland and Bösser, 1993).

Hence, by utilizing the evolution of animals behaviors, the evolutionary algorithm is employed for searching the geomagnetic space. In evolutionary algorithm, each possible solution is defined as the individual, whereas a set of individuals is defined as the population. The evolution method requires maintaining a population of various individuals, according to the operators such as selection, mutation, and so on. The repeated process of recombination, selection, and mutation leads the individuals to adapt to the environment, whereas the selection operator is implemented by a task-oriented evaluation function: the better the AUV performs its task, the more offspring it has. 
However, the local anomalies from the geomagnetic field have significant influences on the proposed geomagnetic navigation system. The next section explains the solution to the anomaly problem.

\section{The Search Algorithm with Geomagnetic Anomaly}

This section discusses how to deal with the local minimum problem within the geomagnetic anomaly areas. Our solution has two phases: the first phase is to determine whether the algorithm falls into the geomagnetic anomaly area, and the second is to determine whether the algorithm arrives at the target location.

The first phase refers to the environmental monitoring operator $\Psi(k)$, which can be expressed as:

$$
\Psi(k)=\frac{\left\|F_{k}^{\prime}-F_{k-1}^{\prime}\right\|}{\frac{1}{\mathrm{~T}_{1}} \sum_{i=1}^{\mathrm{T}_{1}}\left\|F_{k}^{\prime}-F_{k-1}^{\prime}\right\|}=\frac{\left\|F_{k}^{\prime}-F_{k-1}^{\prime}\right\|}{E\left(\Delta F_{\mathrm{T}_{1}}^{\prime}\right)}
$$

where $\mathrm{T}_{1}$ is the period of time, $E(\cdot)$ is described as mean values.

The termination condition in second phase is determined as:

$$
\left\{\begin{array}{c}
\left\|F_{k}^{\prime}-F_{k-1}^{\prime}\right\| \leq \rho \\
F_{k}^{\prime} \leq \varphi
\end{array}\right.
$$

where $\rho$ is a small value zero, $\varphi$ is the scope of the desired target location. The steps for the multi-objective evolutionary algorithm which can address the challenge of the geomagnetic anomaly are given as follows:

Step 1: Population Initialization. Randomly generate $N$ individuals in the population space $Q(Q)$ $\left.\left\{\theta_{j} \mid j=1,2, \cdots, N\right\}\right), \theta$ is the set of samples and given by:

$$
\theta=\left\{\theta_{1}, \theta_{2}, \cdots, \theta_{m}, m=\frac{2 \pi}{\Delta \theta}, \theta_{j}=\Delta \theta \times i, i \in[1, m]\right\}
$$

where $\theta$ is the sampling interval.

Step 2: Individual Selection. Randomly select the sample $\theta_{j}$, and the probability of each individual is given by:

$$
p\left(\theta_{j}\right)=\frac{1}{N}
$$

when the current number of iterations is $k$, the probability of the selected individual $\theta_{i}$ can be obtained as

$$
p\left(\theta_{j}^{k}\right)=\frac{\sum_{j=1}^{N} \delta_{j}\left(\theta_{j}=\theta_{i}\right)}{N}
$$

where $\delta$ is a symbolic function.

Step 3: Population Updating Rules. The termination is calculated if the selected individual accomplishes a successful search. Thus the individual updating rules can be divided into two parts: for one that moves toward the target direction $\left(F_{k}^{\prime} \leq F_{k-1}^{\prime}\right)$, the selected individual heading is reserved in the population. And for the other that moves away from the target direction $\left(F_{k}^{\prime}>F_{k-1}^{\prime}\right)$, the selected individual heading is replaced.

$$
\left\{\begin{array}{c}
\theta_{j}=\theta_{i}, F_{k}^{\prime} \leq F_{k-1}^{\prime} \\
\theta_{j}^{k}=\Delta \theta \times i, F_{k}^{\prime}>F_{k-1}^{\prime}
\end{array}\right.
$$

Step 4: Environmental Monitoring Operator. The monitoring factor $\Psi(k)$ is used to determine whether the algorithm falls into the geomagnetic anomaly area $(\Psi(k) \geq \mu, \mu$ is the threshold value).

Step 5: Motion Constraint Operator. Once the search algorithm is trapped into the local minimum area, the maximum probability $\theta_{i}$ is preserved. This can be expressed as:

$$
\theta_{i}^{k}=\theta_{\max }\left\{p\left(\theta_{i}\right)\right\}
$$

When formula (17) is satisfied, the algorithm jumps out of the local minimum area.

$$
\left\|F_{k+M}^{\prime}-F_{k+M-1}^{\prime}\right\|<\mu E\left(\Delta F_{T_{1}}^{\prime}\right)
$$

Step 6: Termination condition. The navigation is terminated if formula (11) is satisfied. The workflow of the proposed evolutionary algorithm is shown in Figure 2.

\section{NUMERICAL SIMULATIONS}

To verify the effectiveness of the proposed multi-objective evolutionary algorithm, numerical simulations are performed.

\section{Simulation Setup}

The Word Magnetic Model (WMM2010) is used to provide the real time geomagnetic data (Maus et al., 2012). In simulation, a rectangular area is selected from north latitude $30^{\circ}$ and east longitude $100^{\circ}\left(\mathrm{N} .30^{\circ}\right.$ and E. $\left.100^{\circ}\right)$ to north latitude $45^{\circ}$ and east longitude $135^{\circ}$ (N.45 ${ }^{\circ}$ and E. $\left.135^{\circ}\right)$. Considering the non-relevance of the geomagnetic components, only three geomagnetic components are used, which are the north magnetic field $B_{x}$, the east magnetic field $B_{y}$, and the total intensity $B_{F}$, respectively.

Meanwhile, it is assumed that the geomagnetic navigation would encounter a geomagnetic anomaly region. Here, the multimode function is utilized to construct the abnormal geomagnetic environment (see in Figure 3), where the intensity of the highest abnormal geomagnetic field value is $-5,000 n T$.

In Figure 3, the starting position and the target position are depicted by using the three geomagnetic components at $B^{0}=[31,464 n T,-2,036 n T, 52,508 n T]$ and $B^{t}=$ $[26,290 n T,-4,229 n T, 54,254 n T]$, respectively. The circle, "o", stands for the starting position, and the star, “*”, stands for the target position. The related parameters are shown in Table $\mathbf{1 .}$ 


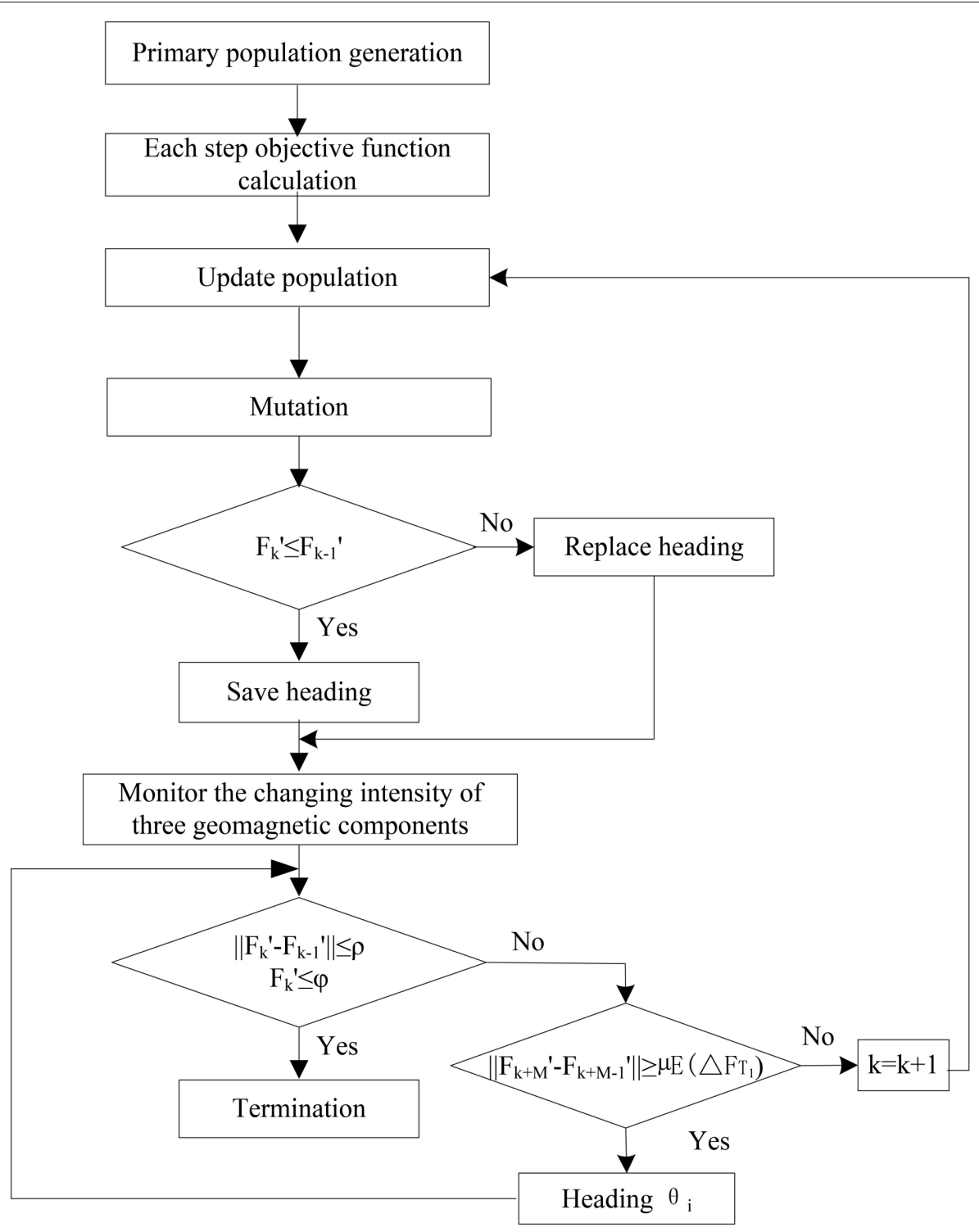

FIGURE 2 | The workflow of the evolutionary search within geomagnetic anomaly.

\section{Simulation Results}

To evaluate the performance of the proposed approach, two cases are evaluated: one occurs without considering geomagnetic anomaly and the other one with geomagnetic anomaly.

Figure 4 illustrates the navigation trajectory without the interference of geomagnetic anomalies, whereas Figure 5 illustrates the moving trajectory with the interference of geomagnetic anomalies. As shown in Figure 5, the previous algorithm is easily trapped into the local minimization in geomagnetic anomaly areas, in which individuals move toward different directions (Liu et al., 2014). It is observed that the navigation performance strongly depends on the geomagnetic anomalies.
It is concluded that the original searching algorithm is not capable for the anomalies of geomagnetic fields, in which AUV moves toward different directions and ultimately fails.

Figure 6 illustrates the proposed approach, where AUV successfully overcomes the influence of the geomagnetic anomalies. During the navigation phase, the individual directions are randomly changed due to the probability selected in the population. Zoomed figure on the left corner shows the two motion constraints, which are performed using the monitoring factor $\Psi(k)$. Two time periods " $c$ " and " $\mathrm{d}$ " are within the geomagnetic anomaly area, while "a" and " $b$ " represent the variation anomalies of the geomagnetic components, respectively. By monitoring the changes of the geomagnetic components, the proposed approach effectively detects the 


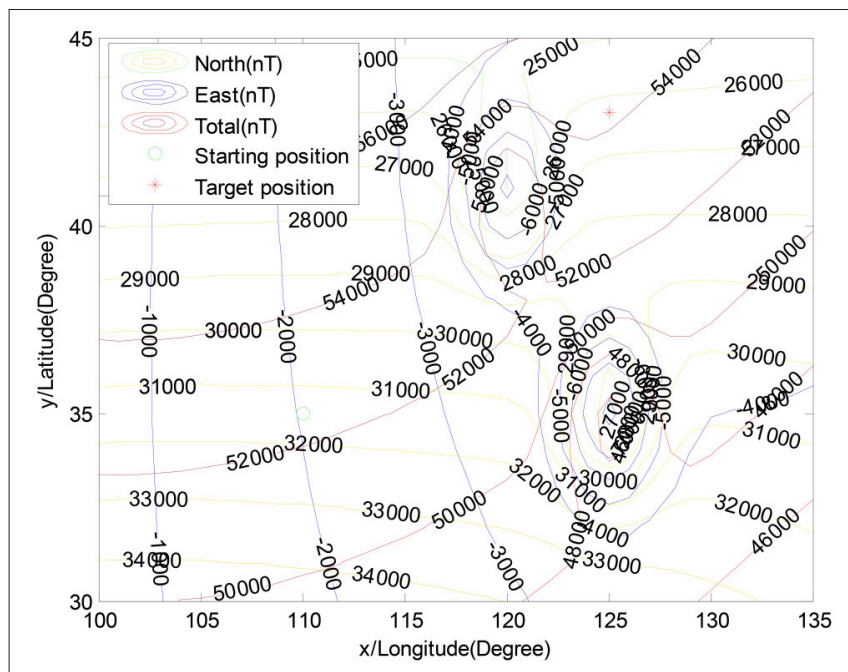

FIGURE 3 | The simulation scenario within multi-modal function.

TABLE 1 | Setting navigation parameters.

\begin{tabular}{llc}
\hline No & Parameters & Size \\
\hline 1 & $L$ & $500 \mathrm{~m}$ \\
2 & $N$ & 50 \\
3 & $\Delta \theta$ & $30^{\circ}$ \\
4 & $\rho$ & 0.007 \\
5 & $\varphi$ & 0.01 \\
6 & $\mu$ & 1.6 \\
7 & $\mathrm{Pm}$ & 0.02
\end{tabular}

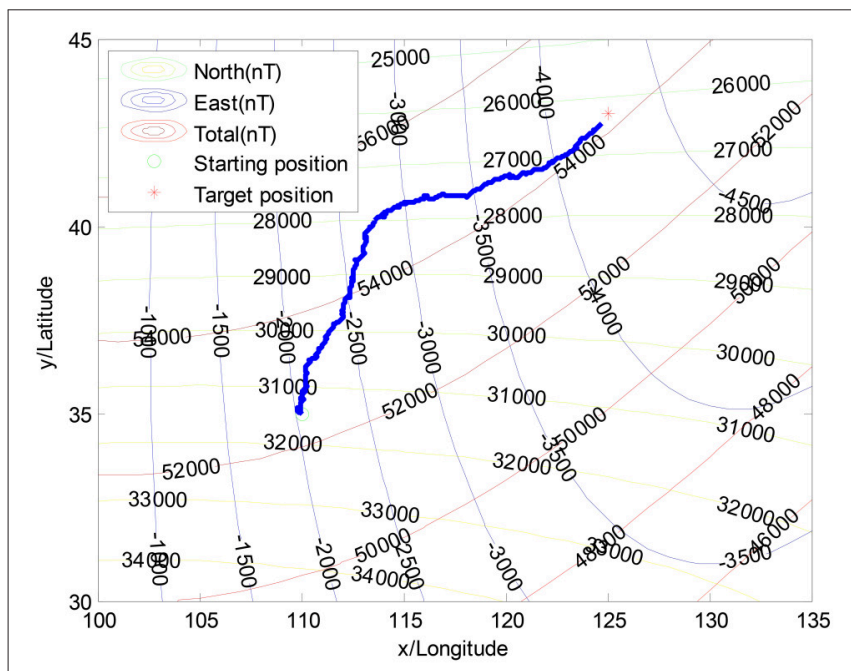

FIGURE 4 | The navigation without the geomagnetic anomalies.

abnormal regions. Then, the statistic characteristics of the convergence state is utilized to the behavior constraints, for the purpose of jumping out the abnormal region.

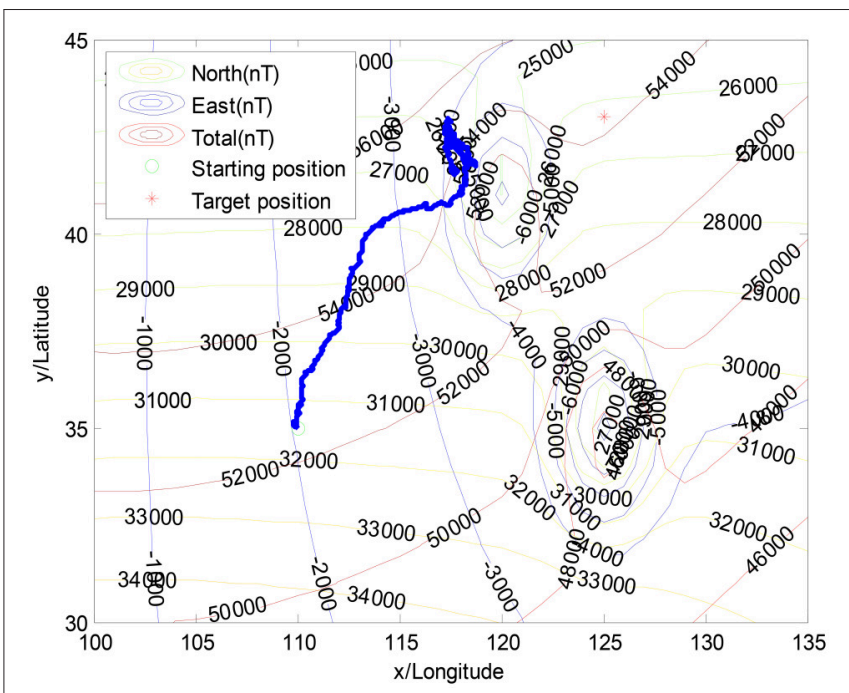

FIGURE 5 | The navigation with the geomagnetic anomalies.

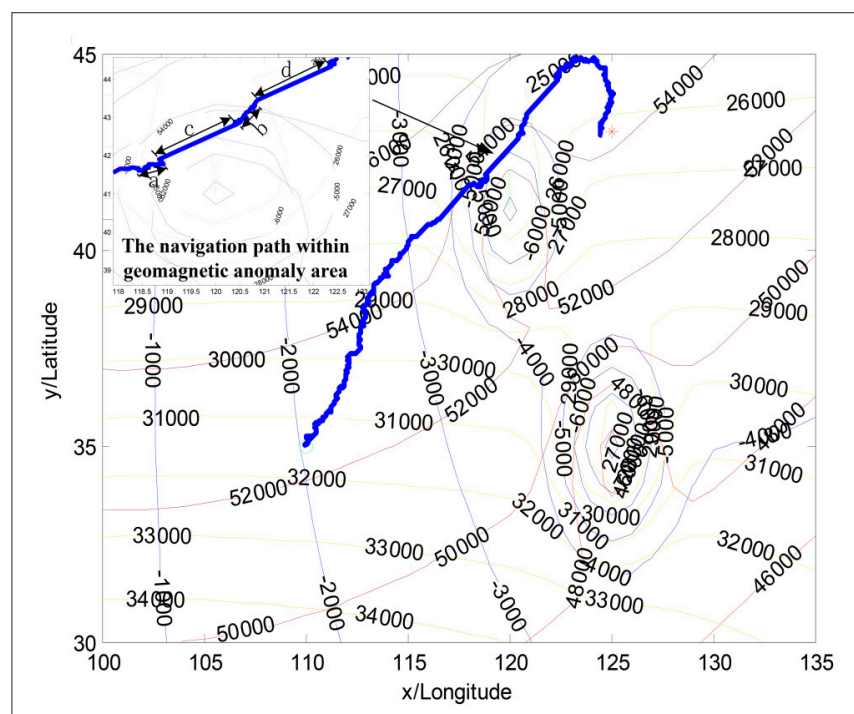

FIGURE 6 | The navigation result in the geomagnetic anomalies with the proposed solution.

\section{Convergence Performance of the Search Algorithm}

The convergence curves of three geomagnetic components are shown in Figure 7. It is observed that the convergence curves present violent shakings in three geomagnetic components.

To better evaluate the performance in presence of geomagnetic anomalies, the convergence properties of three geomagnetic components are provided. As depicted in Figure 8, there is a divergence trend for the three geomagnetic components in periods of "a" and " $b$." The convergence properties of the geomagnetic components are improved after using behavior constraints, demonstrating that the normalized error are able to converge to a stable state. It is observed that the improved 


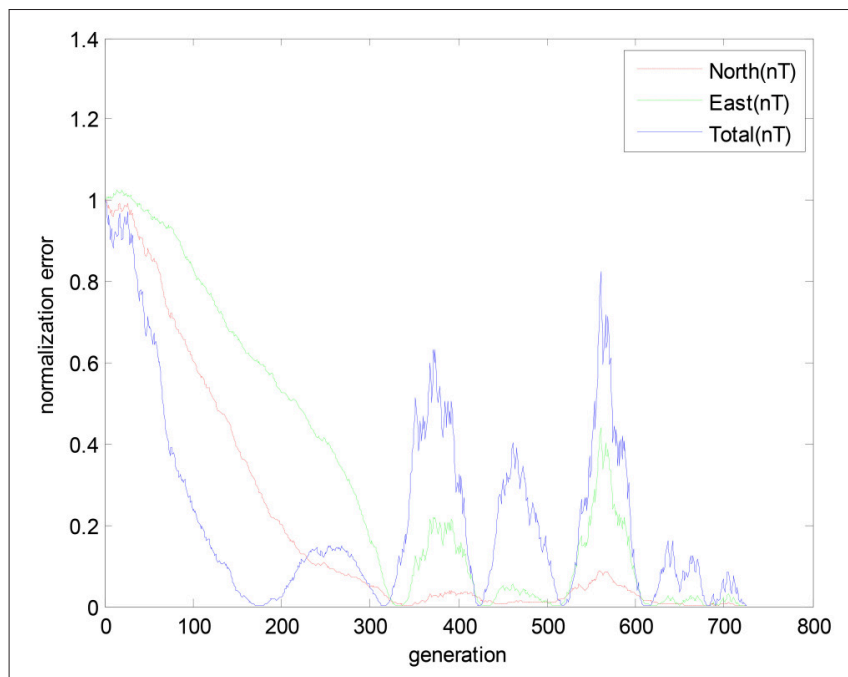

FIGURE 7 | The convergence curves of geomagnetic components within the geomagnetic anomalies.

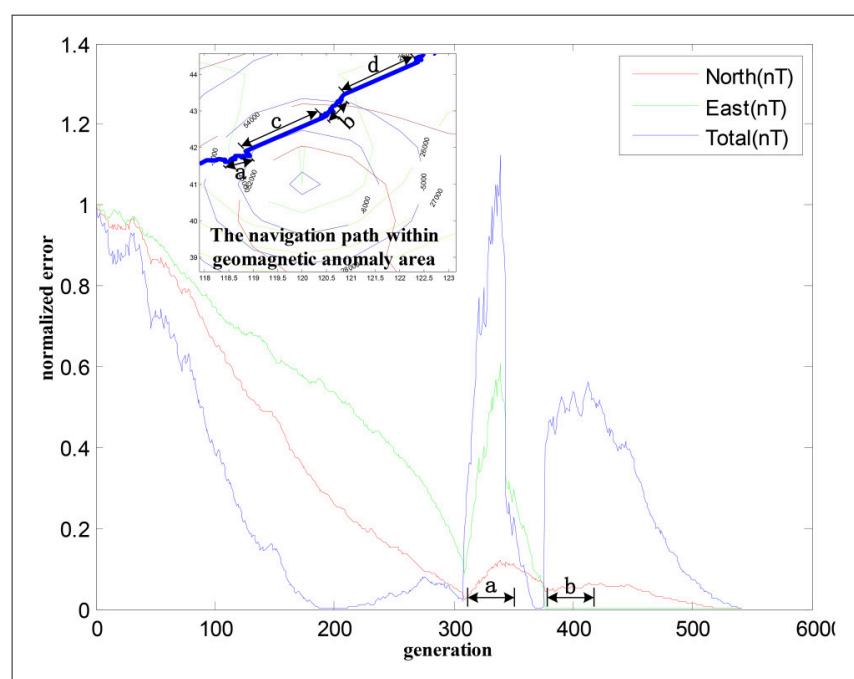

FIGURE 8 | The geomagnetic components convergence within the geomagnetic anomalies.

algorithm could converge the curves of the geomagnetic components to zero in finite steps.

To explore the advantages of the proposed algorithm, the traveling time employed by the AUV in both scenarios is also compared in Figure 9. It shows that the proposed approach could converge to a stable state in scenarios of geomagnetic anomalies, and the navigation process is terminated in 540 steps.

\section{Discussion}

In this paper, the multi-objective evolutionary algorithm has been proposed to address the AUV navigation problem. As a result of geomagnetic anomaly, the distribution of the geomagnetic components is often disrupted over the searching space. Therefore, the environmental monitoring and the behavior constraints are used to tackle the local optimal problem. The

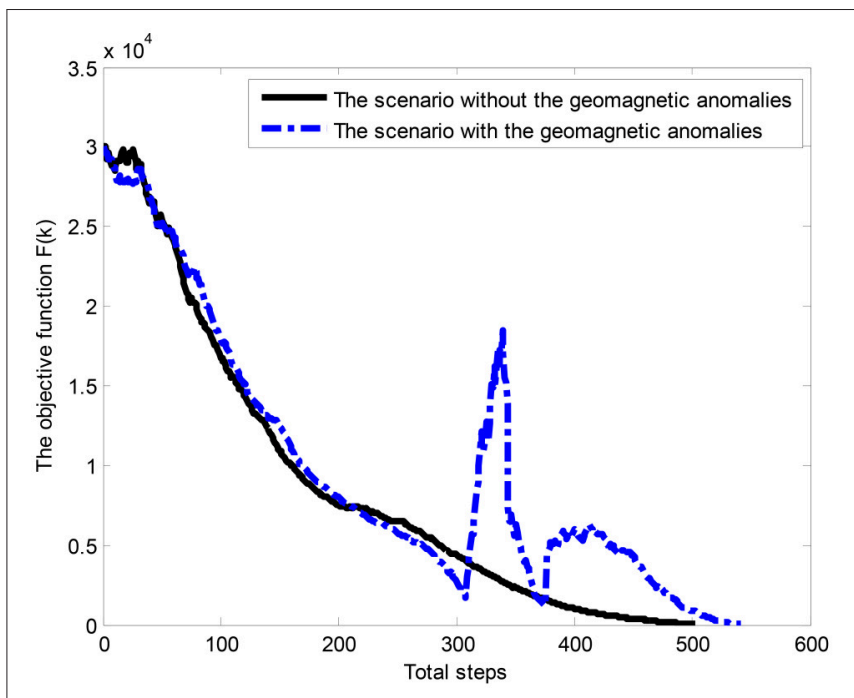

FIGURE 9 | The convergence of the objective function

time complexity of the proposed algorithm is $\mathrm{O}\left(k^{3}\right)$ in the geomagnetic anomalies.

For the geomagnetic navigation, the optimal path between the starting position and the destination is quite challenging without a priori geomagnetic map. Also, the AUV navigation in oceans strongly influences by the geomagnetic anomaly effects. However, the proposed approach indicates that the searching strategy performs optimally for AUV navigation in the geomagnetic anomaly areas.

Thus, the multi-objective searching algorithm with the environmental monitoring and the behavior constraints is proposed to ensure the success and safety of AUV navigation.

\section{CONCLUSION}

This paper presents a novel strategy for bio-inspired geomagnetic navigation in presence of geomagnetic anomalies. Inspired by the biological navigation, in our previous work we proposed an evolutionary schema which helps the AUV to reach the destination without geomagnetic map. However, it suffers the disturbance from geomagnetic anomalies and often converges to the local minimum point. To tackle the problem, this paper proposes an improved navigation model by introducing constraints strategy to make AUV escape from the abnormal regions. Simulation results show that the proposed model effectively overcomes the disturbance of the geomagnetic abnormal for AUV navigation.

\section{AUTHOR CONTRIBUTIONS}

HL wrote the paper and performed the experiments. ML provided some ideas to improve and perfect the paper. FZ reviewed and edited the manuscript. All authors read and approved the manuscript. 


\section{FUNDING}

This work was supported by the National Natural Science Foundation of China (NSFC) under Grants 513 79176.

\section{REFERENCES}

Alerstam, T., Hedenström, A., and Åkesson, S. (2003). Long-distance migration: evolution and determinants. Oikos 103, 247-260. doi: $10.1034 / \mathrm{j} .1600-0706.2003 .12559 . \mathrm{x}$

Bell, W. J. (1992). Searching behaviour: the behaviour ecology of finding resources. J. Anim. Ecol. 61, 370-371.

Boström, J. E., Åkesson, S., and Alerstam, T. (2012). Where on earth can animals use a geomagnetic bicoordinate map for navigation? Ecography 35, 1039-1047. doi: $10.1111 / j .1600-0587.2012 .07507 . x$

Caifa, G., Anliang, L., Hong, C., and Huabo, Y. (2011). Algorithm for geomagnetic navigation and its validity evaluation. Int. Conf. Comput. Sci. Autom. Eng. 1, 573-577. doi: 10.1109/CSAE.2011.5953286

Caiti, A., Di Corato, F., Fenucci, D., Ridolfi, A., Pugi, L., Monni, N., et al. (2014). Experimental results with a mixed USBL/LBL system for AUV navigation. Underwater Commun. Netw. 2014, 1-4. doi: 10.1109/UComms.2014.7017129

Chong, K., Zhang, X., and Fan, L. (2014). Geomagnetic-matching aided navigation based on ICCP algorithm. J. Basic Sci. Eng. 22, 598-605. doi: $10.3969 /$ j.issn.1005-0930.2014.03.018

Cliff, D., Husbands, P., and Harvey, I. (1993). Explorations in evolutionary robotics. Adaptive Behav. 2, 73-110. doi: 10.1177/105971239300200104

Dennis, T. E., Rayner, M. J., and Walker, M. M. (2007). Evidence that pigeons orient to geomagnetic intensity during homing. Proc. R. Soc. Lond. B Biol. Sci. 274, 1153-1158. doi: 10.1098/rspb.2007.3768

Droste, S., Jansen, T., and Wegener, I. (2002). On the analysis of the $(1+1)$ evolutionary algorithm. Theor. Comp. Sci. 276, 51-81. doi: 10.1016/S0304-3975(01)00182-7

Fu-qing, P. (2006). Geomagnetic model and geomagnetic navigation. Hydrogr. Surv. Chart. 2, 73-75. doi: 10.3969/j.issn.1671-3044.2006.02.021

Goldenberg, F. (2006). Geomagnetic navigation beyond the magnetic compass. Proc. IEEE/ION Plans 2001, 684-694. doi:10.1109/PLANS.2006.1650662

Gould, J. L. (1984). Magnetic field sensitivity in animals. Annu. Rev. Physiol. 46, 585-598. doi: 10.1146/annurev.ph.46.030184.003101

Hao, Y. L., Zhao, Y. F., and Jun-Feng, H. U. (2008). Preliminary analysis on the application of geomagnetic field matching in underwater vehicle navigation. Prog. Geophys. 23, 594-598. doi: 10.6038/pg20080601

Hays, G. C. (2013). Animal navigation: salmon track magnetic variation. Curr. Biol. 23, R144-R145. doi: 10.1016/j.cub.2013.01.025

Jia, L., Wang, Y. G., Shan, B., and Yan, T. (2012). Simulation of single element geomagnetic matching navigation based on intensified MAD. Mod. Def. Technol. 1, 90-94. doi: 10.3969/j.issn.1009-086x.2012.01.019

Kimchi, T., Etienne, A. S., and Terkel, J. (2004). A subterranean mammal uses the magnetic compass for path integration. Proc. Natl. Acad. Sci. U.S.A. 101, 1105-1109. doi: 10.1073/pnas.0307560100

Klinowska, M. (1986). Cetacean live stranding dates relate to geomagnetic disturbances. Aquat. Mammals 11, 109-119.

Liu, M., Li, H., and Liu, K. (2014). Geomagnetic navigation of AUV without a priori magnetic map[C]//OCEANS 2014-TAIPEI. IEEE 2014, 1-5. doi: 10.1109/ OCEANS-TAIPEI.2014.6964431

Liu, M., Liu, K., Yang, P., and Lei, X. (2013). Bio-inspired navigation based on geomagnetic robotics and biomimetics (ROBIO). IEEE International Conference on IEEE 2013 (Shenzhen), 2339-2344.

Lohmann, K. J. (2010). Animal behaviour: magnetic-field perception. Nature 464, 1140-1142. doi: $10.1038 / 4641140 \mathrm{a}$

Lohmann, K. J., Lohmann, C. M. F., and Putman, N. F. (2007). Magnetic maps in animals: nature’s GPS. J. Exp. Biol. 210, 3697-3705. doi: 10.1242/jeb.001313

Maus, S., Macmillan, S., McLean, S., and Hamilton B. (2012). The US/UK World Magnetic Model for 2010-2015. Available online at: https://www.ngdc.noaa.gov/ geomag/WMM/DoDWMM.shtml

\section{ACKNOWLEDGMENTS}

The authors are also grateful for the constructive suggestions from reviewers that significantly enhance the presentation of this paper.

McFarland, D., and Bösser, T. (1993). Intelligent Behavior in Animals and Robots. MIT Press.

Paolo, L., Hays, G. C., and Floriano, P. (2003). A review of long-distance movements by marine turtles, and the possible role of ocean currents. Oikos 103, 293-302. doi: 10.1034/j.1600-0706.2003.12123.x

Peng, X., and Wu, Y. (2017). Large-scale cooperative co-evolution using nichingbased multi-modal optimization and adaptive fast clustering. Swarm Evol. Comput. doi: 10.1016/j.swevo.2017.03.001. [Epub ahead of print].

Peng, X., Liu, K., and Jin, Y. (2016). A dynamic optimization approach to the design of cooperative co-evolutionary algorithms. Knowledge-Based Syst. 109, 174-186. doi: 10.1016/j.knosys.2016.07.001

Schulten, K. (1982). "Magnetic field effects in chemistry and biology," in Festkörperprobleme 22, eds K. Schulten, H. Staerk, and A. Weller (Berlin Heidelberg: Springer), 61-83.

Shen, C., Shi, Y., and Buckham, B. (2017). Integrated path planning and tracking control of an AUV: a unified receding horizon optimization approach. IEEE/ASME Trans. Mech. 22, 1163-1173. doi: 10.1109/TMECH.2016.2612689

Shi, Y., Shen, C., Fang, H., and Li, H. (2017). Advanced control in marine mechatronic systems: a survey. IEEE/ASME Trans. Mech. 22, 1121-1131. doi: $10.1109 /$ tmech.2017.2660528

Talwani, M. (1965). Computation with the help of a digital computer of magnetic anomalies caused by bodies of arbitrary shape. Geophysics 30, 797-817. doi: 10.1190/1.1439654

Teixeira, F., and Pascoal, A. (2008). "Geophysical navigation of autonomous underwater vehicles using geomagnetic information," in Proceedings of NGCUV'08-IFAC Workshop on Navigation, Guidance and Control of Underwater Vehicles (Ireland).

Vrugt, J. A., and Robinson, B. A. (2007). Improved evolutionary optimization from genetically adaptive multimethod search. Proc. Natl. Acad. Sci.U.S.A. 104, 708-711. doi: 10.1073/pnas.0610471104

Wadhams, P. (2012). The use of autonomous underwater vehicles to map the variability of under-ice topography. Ocean Dyn. 62, 439-447. doi: 10.1007/s10236-011-0509-1

Winklhofer, M. (2009). The physics of geomagnetic-field transduction in animals. IEEE Trans. Magn. 45, 5259-5265. doi: 10.1109/TMAG.2009.2017940

Wynn, R. B., Huvenne, V. A. I., Le Bas, T. P., Murton, B. J., Connelly, D. P., Bett, B. J., et al. (2014). Autonomous underwater vehicles (AUVs): their past, present and future contributions to the advancement of marine geosciences. Mar. Geol. 352, 451-468. doi: 10.1016/j.margeo.2014.03.012

Xie, W., Qu, Z., and Li, Q. (2013). A fast algorithm of the geomagnetic correlation matching based on MSD. Adv. Intell. Syst. Res. 45, 59-62. doi: 10.2991/case-13.2013.15

Yi, L., Lei, Y., and Tong, Q. (2008). "Underwater geomagnetic navigation based on ICP algorithm," in IEEE International Conference on Robotics and Biomimetics (Sanya), 2115-2120.

Zhou, J., Ge, Z., Shi, G., and Liu, Y. (2008). Key technique and development for geomagnetic navigation. J. Astronaut. 5, 1467-1472. doi: 10.3873/j.issn.10001328.2008.05.001

Conflict of Interest Statement: The authors declare that the research was conducted in the absence of any commercial or financial relationships that could be construed as a potential conflict of interest.

Copyright (c) $2017 \mathrm{Li}$, Liu and Zhang. This is an open-access article distributed under the terms of the Creative Commons Attribution License (CC BY). The use, distribution or reproduction in other forums is permitted, provided the original author(s) or licensor are credited and that the original publication in this journal is cited, in accordance with accepted academic practice. No use, distribution or reproduction is permitted which does not comply with these terms. 